\section{Bonded states}

Metal-to-Metal Bonded States of the Main Group Elements. By M. J. Taylor. Pp. 211. (Academic: New York and London, May 1975.) \$15.25; £5.80.

Compounds in which ligands are linked to a cluster of atoms, instead of to a single atom, have opened up much novel chemistry in recent years. Much of this has been in transition metal chemistry, but cluster compounds spread right across the periodic table, and interest in them stems from their novel compositions, the apparently low valence states of the elements, their stereochemistry and their valence-theoretical description.

Dr Taylor has explicitly restricted himself to compounds of the main group elements. In addition, he limits consideration to compounds of elements that, in the elementary state are regarded as metals. There is no real justification for doing so, and the arbitrary restriction has some illogical consequences. Thus, he has to discuss the cationic clusters formed by selenium and tellurium without proper reference to their exact analogues in sulphur chemistry, and he makes no mention at all of the anionic clusters formed by silicon and germanium. But equally mistakenly in my opinion he broadens his survey to include all compounds in which two 'metal' atoms are linked. The real interest of metal-metal bonding surely resides in the consequence of electron delocalisation within a cluster or chain of identical (or at least essentially interchangeable) atoms. The pro-

\section{Literature on water}

Water and Aqueous Solutions: Introduction to a Molecular Theory. By Arieh Ben-Naim. Pp. xvi+474. (Plenum: New York and London, 1974.) \$34.90.

THE basic thesis of this book is that some sort of mixture model provides a fundamentally correct physical description of the thermodynamic properties of water and aqueous solutions and that mixture models can be made respectable or rigorous by embedding them in or deriving them from the fundamental statistical mechanical theory of fluids. The author is not particularly concerned with establishing the validity of any particular mixture model. Rather, he is concerned with the features common to all such models and with giving precise statistical mechanical definitions of the concepts used in discussing them.

The first half of the book discusses the statistical mechanics of fluids, molecular distribution functions, and the theory of solutions. The last half is concerned with liquid water, water with one simple (non-hydrogen-bonding) solute, and perties of, for example, the $\left(\mathrm{CH}_{3}\right)_{3} \mathrm{Sn}$ group, like those of the $\left(\mathrm{CH}_{3}\right)_{3} \mathrm{Si}$ or even the $\left(\mathrm{CH}_{3}\right)_{3} \mathrm{C}$ group, are those of a straightforward $\sigma$-bonding group; there is nothing special about such compounds as $\left(\mathrm{CH}_{3}\right)_{3} \mathrm{SnCo}(\mathrm{Co})_{4}$, or $\left(\mathrm{CH}_{3}\right)_{6} \mathrm{Sn}_{2}$, to justify special attention to the "metalmetal' bond. The amount of space devoted to compounds of this kind dilutes what should be the main interest of the book.

What the book does provide is much information on the formation of dimer and cluster species, in solutions and melts, and structural information based on vibrational spectroscopy and crystal structure. It has a thorough bibliography, heavily biased towards work of the last 15 years, including references up to 1973 . This review content is its main value. For student reading, it does not bring a unifying outlook to a diverse field; for example, there is repeated terminological confusion between the oxidation states, which may often be experimentally determined, and the valence states of elements which in cluster compounds are certainly different from the oxidation states and which may be debatable. There are many typographical errors, but few will lead to confusion. Readers will note that, on the dust jacket, Academic Press have now decreed that selenium and tellurium shall be Group Vb elements.

The author states that the book is an expanded treatment of a review. One must query whether that expansion was really justified. A good review does not necessarily produce an equally good monograph.

J. S. Anderson

water with two simple solutes. The emphasis in this second half is almost entirely on thermodynamic properties, rather than on time dependent or spectroscopic measurements. A major limitation of the book (which is a reflection of when its writing was completed) is that it contains only a brief discussion of the earliest computer simulation studies of realistic models of water. Such studies should provide the most convincing evidence for or against the usefulness of mixture models.

Many of the features of the book are of interest and of value even to the reader who does not share the author's preference for mixture models; for example, the careful discussion of those aspects of solution theory which are needed to understand experiments on aqueous solutions, the precise discussion of standard thermodynamic quantities of transfer, and a statistical mechanical definition of the concept of hydrophobic interaction. Though the book does not answer many questions it does formulate many about water and aqueous solutions in a precise way. It is a useful companion volume for the large recent review literature on water.

Hans C. Andersen

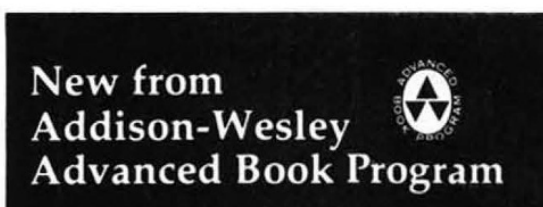

LECTURE NOTES AND SUPPLEMENTS IN PHYSICS SERIES

David Pines, Editor

Publication resumed with No. 14 Hans Frauenfelder and Ernest M. Henley NUCLEAR AND PARTICLE PHYSICS A: Background and Symmetries

The first part of a graduate-level introduction to particle and nuclear physics, stressing concepts and symmetry laws. Most of the necessary background is briefly developed; however, a knowledge of quantum mechanics and electrodynamics is assumed. The authors have selected topics that fill a gap between an introductory text and research reviews.

Contents: Historical Survey and Introduction. Classical Concepts and Conservation Laws. Particles, Antiparticles and Fields. Nonclassical Conservation Laws. Superposition and Interference. Appendices. Index. 1975, xviii, 572 pp., illus., hardbd. $£ 11.85$; paperbd. $£ 7.45$

\section{FRONTIERS IN PHYSICS SERIES}

David Pines, Editor

No. 47:

\section{HYDRODYNAMIC FLUCTUATIONS} BROKEN SYMMETRY, AND CORRELATION FUNCTIONS Dieter Forster

Presents the theory of fluctuations and linear response in many-body systems and their description in terms of response functions. The emphasis is to formulate the theory in a simple and unified fashion to allow the reader to appreciate and exploit the many analogies which exist between, for example, superfluids and liquid crystals. The level of the text is deliberately held very simple. It will be easily accessible to physics and chemistry students in their first or second year of graduate school, particularly because the emphasis is on the physical principles rather than on mathematical completeness and detail.

Contents: Introduction. A Simple ExampleSpin Diffusion. Formal Properties of Correlation Functions. The Normal Fluid. The Memory Function Formalism. Brownian Motion. Broken Symmetry. Hydrodynamic Spin Waves in Ferromagnets. Hydrodynamic Spin Waves in Antiferromagnets. Superfluids. Nematic Liquid Crystals. Superconductors. Appendix. References. Index. 1975, xx, 326 pp., hardbd., $£ 10.75$; paperbd. $£ 6.35$

\section{New Serial Publication}

PHYSICS OF QUANTUM ELECTRONICS

S. F. Jacobs, M. Sargent III, and M. O. Scully, Editors

Vol. 1: High Energy Lasers and Therr Applications 1974 , xi, 411 pp., illus., $£ 10.75$ hardbd

Vol. 2: Laser Applications to Optics and Spectroscopy 1975 , xii, 510 pp., illus., $£ 12.95$ hardbd.

Edited by S. F. Jacobs, M. Sargent III, M. O. Scully, J. F. Scott

Detailed contents available on request. Continuation orders invited.

Addison-Wesley Publishers, Ltd.

West End House Advanced Book Program 11 Hills Place $\quad$ Reading, Mass. 01867 London U.S.A 